\title{
Research on energy efficient fusion-driven routing in wireless multimedia sensor networks
}

\author{
Kai Lin ${ }^{1 *}$ and Min Chen ${ }^{2}$
}

\begin{abstract}
The limitation of energy supply is a crucial problem in wireless multimedia sensor networks. In this article, we research and optimize the energy utilization during data collection by adopting mobile agent nodes. First, we demonstrate that the energy consumption of the whole network is affected by the data correlation coefficient for a multimedia sensor network with a random node deployment. Then, we give the method to find the optimized position of mobile agent node for maximizing the energy-saving efficiency by fusion process. Finally, we propose an energy efficient fusion-driven routing (EEFR) based on cluster hierarchy. To obtain a better performance, the cluster structure is divided based on square grid topology. Extensive simulation experiments have been made to evaluate our proposed EEFR with several performance criteria. The results show that EEFR can effectively save energy consumption of network, and the consumption is relatively balanced.
\end{abstract}

Keywords: wireless multimedia sensor networks, energy efficiency, data fusion, cluster hierarchy, fusion-driven routing

\section{Introduction}

Wireless multimedia sensor networks (WMSNs) have been the target of active research with a particular emphasis on high-caliber information collection. Recent rapid development in the sensor, wireless network, multimedia and embedded computing areas now make it possible to deploy a large number of multifunctional and inexpensive multimedia sensor nodes to achieve high quality data acquisition [1]. In general, the sensor nodes of WMSNs are equipped with CMOS camera, microphone, and other kinds of sensors for achieve the fine-grained, accurate information in a comprehensive environmental monitoring. Compared with traditional WMSN, WMSNs can capture the surrounding environment in a variety of media information and has outstanding performance in multimedia signal acquisition and processing. It not only can enhance existing multimedia sensor network applications, but also enable several new applications, such as multimedia surveillance multimedia sensor networks, advanced health care

\footnotetext{
* Correspondence: link@dlut.edu.cn

'School of Computer Science and Engineering, Dalian University of Technology, Dalian, Liaoning, China

Full list of author information is available at the end of the article
}

delivery, industrial process control, mobile multimedia sensor networks [2] and so on [3,4].

These sensor nodes are typically lightweight with limited battery capacity, processing power and communication bandwidth. In order to ensure the normal operation of WMSNs, the energy consumption of communication must be maintained in minimum level. During the network operation, data fusion should be considered to eliminate unnecessary communications since the massively deployed sensor nodes generate a huge amount of redundant data, which makes it critical to collect only the valuable data.

In traditional fusion architecture of WMSNs, all the sensory data have the same structure and need to be fused by the routing nodes before sent to the sink node. However, for some complicate phenomena, it is a trend for WMSNs to be transferred from homogeneous to heterogeneous, wherein nodes are equipped with various sensors in the heterogeneous network to monitor multitargets separately or cooperatively. The heterogeneous network increases the complexity of fusion process but decrease the data correlation coefficient that is used to examine the relationship strength between sensory data, both of them will consume the energy resources additionally [5]. 
Table 1 Parameters in simulation

\begin{tabular}{ll}
\hline Parameter & Value \\
\hline Initial energy & $20 \mathrm{~J}$ \\
Distribution density & $0.003 / \mathrm{m}^{2}$ \\
Energy consumption/circuit & $50 \mathrm{~nJ} / \mathrm{bit}$ \\
Energy consumption of amplifier & $d<87 \mathrm{~m} 10 \mathrm{pJ} / \mathrm{bit} \mathrm{\textrm {m } ^ { 2 }}$ \\
Network area & $9 \times 10^{4} \mathrm{~m}^{2}$ \\
Bandwidth & $1 \mathrm{Mbps}$ \\
Of data fusion & $15 \mathrm{~nJ} / \mathrm{bit}$ \\
Of amplifier & $d \geq 87 \mathrm{~m} 10 \mathrm{pJ} / \mathrm{bit} \mathrm{m}^{2}$ \\
\hline
\end{tabular}

In this article, we study the data fusion with the consideration of data attribute difference in heterogeneous network with relatively high node density. Thus, cluster hierarchical structure is exploited in the network because of its effectiveness of distributed management in a largescale network. In addition to the use of data fusion to alleviate the strong correlativeness of sensory data among the sensor nodes located in one cluster. Different with the sensor node hosting agent (soft agent) [6], the mobile agent node in our research has unique physical structure, it can freely move and is not limited by energy supply. Hence, the mobile agent node is introduced to act as the cluster head because of its much heavier tasks than the ordinary sensor nodes. Thus, a kind of energy efficient cluster routing protocol is proposed by the use of the mobility of agent node. The main contributions of this article are summarized as follows:

- Compared to the traditional cluster routing schemes with the intrinsic feature of scalar data collection, the proposed algorithm emphasizes the interaction among different multimedia data attributions. Thus, we systemically analyze the data collecting characterization in a heterogeneous environment with cluster hierarchy. Then, the effect of multiattribute-based data fusion regarding energy consumption is analyzed.

- Based on our analysis of the data collecting characterization, an energy efficient fusion-driven routing (EEFR) scheme is proposed to maximize the efficiency of fusion by the mobility of agent node. Moreover, the remaining energy of node is also considered during data collection to balance energy consumption.

The rest of this article is organized as follows: Section 2 states related works. Section 3 gives the system models and problems. Section 4 analyzes energy efficiency with fusion process. Section 5 presents EEFR hierarchy. Simulation results are provided in Section 6. Finally, Section 7 concludes the article.

\section{Related work}

With the development of WMSNs, more and more attentions are focused on improving the network efficiency and saving energy consumption by data fusion process combined with different applications. Currently, the relative research includes data fusion mechanism, cluster routing, and mobility of agent.

Rickenbach et al. proposed an optimal algorithm MEGA for foreign-coding and an approximating algorithm LEGA for self-coding in [7]. In MEGA, each node sent raw data to its encoding point using directed minimum spanning tree, and encoded data were then transmitted to the sink through SPT. Krishnamachari et al. investigated the impact of data aggregation on these networking metrics by surveying the existing data aggregation protocols in [8]. Goel et al. proposed LEGA using shallow light tree as the data gathering topology in [9]. Lin et al. investigated the process and performance of multi-attribute fusion in data gathering, and then proposed a self-adaptive threshold method to balance the different change rates of each attributive data. They presented a method to measure the energy-conservation efficiency of multi-attribute fusion and designed a novel energy equilibrium routing method, multi-attribute fusion tree [5]. Luo et al. developed an online algorithm capable of dynamically adjusting the route structure when sensor nodes joined or left the network in [10]. Furthermore, by only performing such reconstructions locally and maximally preserving existing routing structure, the online algorithm could be readily implemented in real networks in a distributed manner and promised extremely small performance deviation from the off-line version and outperformed other routing schemes with static aggregation decision. Motivated by the limitation of minimum fusion Steiner tree, they designed a novel routing algorithm, called adaptive fusion Steiner tree for energy efficient data gathering in multimedia sensor networks. Anandkumar et al. presented a novel formulation for optimal sensor selection and in-network fusion for distributed inference known as the prize-collecting data fusion in terms of optimal trade-off between the costs of aggregating the selected set of sensor measurements and the resulting inference performance at the fusion center in [11]. Wun et al. presented a novel system for decoupling the process of semantic data fusion from application logic based on semantic Content-based Publish/ Subscribe techniques [12].

Many cluster routing have been developed to optimize the energy consumption of network. Heinzelman et al. proposed a low energy adaptive clustering hierarchy $(\mathrm{LEACH})$ in [13]. Since then, the clustering routing plays an important and essential role in the routing. However, LEACH cannot guarantee either the position 
or the number of clusters in the network. Besides, it does not fully consider the energy of sensor nodes during the selection of cluster head nodes. Wang et al. proposed SoRCA to implement self-healing, but it partitions the working area into fixed hexagons, and considers each hexagon to be fully covered if there is one active node within the cell in [14]. Xu et al. proposed GAF to divide the coverage area into squares and considers the nodes in a square to be equivalent for routing in [15]. Lin et al. proposed an adaptive reliable routing based on clustering hierarchy named $\mathrm{ARCH}$, which included energy prediction and power allocation mechanism. To obtain a better performance, the cluster structure was formed based on cellular topology. The introduced prediction mechanism makes the sensor nodes predicted the remaining energy of other nodes, which dramatically reduced the overall information needed for energy balancing [16]. The research work only was focused on the reliable transmission while the data attribute and fusion process were not considered. Liu and Chang et al. proposed GAF-h and ZBP to take the advantage of hexagon like cellular in stead of square, but they are not suitable in random deployment of nodes in practice in $[17,18]$. These four cluster routings only consider the position of sensor nodes, while ignore the energy level of the candidate cluster head node. Besides, each sensor node has to know its accurate position to form the cellular structure. It cannot meet the requirement of low cost. Lin et al. proposed a clustering hierarchy based on cellular topology (CHCT), in which the remaining energy and position of sensor nodes are simultaneously considered during the cluster structure construction, and the desired cluster structure is generated even in the case of nodes without locating device [19]. Wu et al. explore the theoretical aspects of the nonuniform node distribution strategy that addresses the energy hole problem. They propose a distributed shortest path routing algorithm tailored for the proposed non-uniform node distribution strategy [20].

Many moving or QoS provisioning strategies for sink node or agent are presented to optimize the energy consumption; however, they can only support the data collection based on inquiry but not reduce the energy load [21-24]. Wang et al. changed the station mobility model to linear plan for the optimized station mobility and special stop point [25]. Shah et al. presented a data mules, which can complete the data transmission by mules mobility [26]. The load in this method is small but the real time of data cannot be guaranteed. Wang et al. presented a data gathering model by agent mobility, where the station is stable and the agents distributed among the station are moving around the circle [27]. This method cannot solve the node load balance over two hops. Gandham et al. analyzed this problem and presented a combining model with data routing and station mobility to reach a load balancing [28].

\section{System model and problem statement \\ A. System model}

Network model: In this article, we adopt a WMSN formed by $n$ randomly deployed sensor nodes, denoted by $S=\left\{s_{1}, s_{2}, \ldots, s_{n}\right\}$ and only one static sink node acts as the destination of the whole network. All the sensor nodes are used for data collection in the monitoring area and keep stable after the deployment. In addition, for improving the performance of WMSNs, we also introduce $m$ mobile agent nodes, denoted as $G=\left\{g_{1}, g_{2}\right.$, ..., $\left.g_{m}\right\}$, where $m$ is far less than $n$ due to their higher cost. These agent nodes can control their moving traces and are responsible for collecting the sensory data generated by the surrounded sensor nodes, then relay to the sink node. The main distinguished features of the system are as the followings:

- The sensor nodes are not equipped with the same kinds of sensors.

- All the agent nodes are movable and not limited by energy. They have a much better ability of communication and computation than sensor nodes.

- The sink node is not limited by energy and has highest ability of communication and computation.

- Sensor nodes can adjust the transmission power to save energy and the communication links are symmetrical, where the distance from the receiver to the transmitter can be calculated by the intensity of the received signal.

- All the sensor nodes have the same initial energy and the capacity of computing and communication.

We focus only on the communications among the sensor nodes, the mobile agents, and the sink node, whereas the communications between the sink node and devices outside the network are out of the scope of this article.

Fusion model: Data fusion is adopted to reduce the redundant sensory data during data collection. The employed data fusion model in our research is similar to reference [10]. In such model, for any attribute, $p$, when a node $s_{i}$ receives the data sent from node $s_{j}$, the total data amount after fused with the data generated by itself is expressed as

$$
\begin{aligned}
\widetilde{D}\left(s_{i}, p\right)= & \max \left(D\left(s_{i}, p\right), D\left(s_{j}, p\right)\right) \\
& +\min \left(D\left(s_{i}, p\right), D\left(s_{j}, p\right)\right)(1-\sigma),
\end{aligned}
$$

where $D\left(s_{i}, p\right)$ and $D\left(s_{j}, p\right)$ represent the data amount of attribute $p$ generated by node $s_{i}$ and $s_{j}$, respectively. $\sigma$ represents the data correlation coefficient between node 
$s_{i}$ and $s_{j}$. From this equation, it can be seen that the higher $\sigma$ can generate less data amount.

Energy model: We assume that all the nodes have the same initial energy while only the sink node and mobile agent node are not limited by energy supply. Similar to [19], the energy spent by transmitting 1 bit data over distance $d$ is $e_{t}(d)=\varepsilon_{\text {elec }}+\varepsilon_{\text {amp }} \cdot d^{k}$, where $\varepsilon_{\text {elec }}$ is the energy spent by transmitter electronics, $\varepsilon_{\mathrm{amp}}$ is the transmitting amplifier, and $k(k \geq 2)$ is the propagation loss exponent. $\varepsilon_{\text {elec }}$ and $\varepsilon_{\text {amp }}$ are both system parameters. The corresponding energy dissipation in data reception is $e_{r}=\varepsilon_{\text {elec }}$. In addition, although data fusion can reduce the energy consumption, it still introduces the extra energy consumption, where each fusion for 1 bit data is denoted by $e_{f}$.

\section{B. Problem statement}

Now we begin to formulate the problem. As shown in Figure 1, the multimedia sensor network consists of three parts: a large amount of sensor nodes, some mobile agent nodes, and one sink node. These mobile agent nodes are uniform distributed in the network, then the whole network is divided into some clusters. Each cluster has one agent node act as cluster head which needs to manage the data collection in its cluster. According to different task requirements, sensor nodes complete data collection and send the sensory data to agent node in their cluster, then the agent node relays these data to the sink node. To meet the requirements of different tasks, the network is carried with a series of various sensors. Each kind of sensor can generate data with different attributes. We assume that there are $t$ kinds of sensors, then there are $t$ kinds of sensory data with different attributes. Each sensor node is equipped with $q(1 \leq q \leq t)$ kinds of the sensors.

Data collection is divided into two phases, one is intra-cluster collection and the other one is inter-cluster

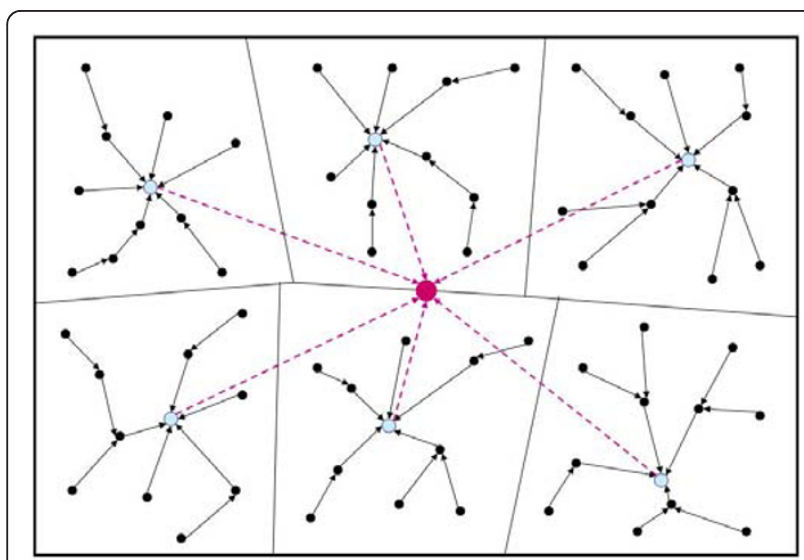

Figure 1 Data collection with mobile agent. collection. For the intra-cluster collection, the sensor nodes are the source and the agent node is the destination. For the inter-cluster collection, the agent node is the source and the sink node is the destinations. As the communication capacity of sensor node is limited, most of them cannot directly transmit the data to the mobile agent node; hence multi-hop transmissions are necessary to solve this problem. For avoiding the data loss, each sensor node needs to establish at least one path to the mobile agent in its cluster.

Definition 3.1 Data collection space: Let $D_{p}$ be the data set that complete the $p$ kind of task for data collection. Each kind of task corresponds to one kind of data set, and we assume there are $k$ kinds of tasks. Then, the generated $k$ kinds of data sets compose the data collection space, denoted as $\widehat{D}=D_{1} \times D_{2} \times \cdots \times D_{k}$.

Definition 3.2 Efficient data fusion: Only those fusion processes that can reduce the energy consumption of network are regarded as efficient data fusion.

Definition 3.3 Energy efficiency: Energy efficiency refers to complete the task with the least energy while the energy consumption is more balanced.

As mobile agent node is not limited by energy supply, hence only the data collection of inter-cluster is considered. Our optimization object is to design a routing protocol that can guarantee energy efficiency while delivering data from all source nodes to the sink node in its cluster $S$. This problem can be formulated as follows:

$$
\left\{\begin{array}{l}
\min \sum_{s_{i}, s_{j} \in S} e\left(s_{i}, s_{j}\right) x\left(s_{i}, s_{j}\right) \\
\text { s.t. for } \forall s_{j} \in S, \sum x\left(s_{i}, s_{j}\right)=1,
\end{array}\right.
$$

where $x\left(s_{i}, s_{j}\right)$ represents whether a connection exists between node $s_{i}$ and $s_{j}$. If node $s_{j}$ is the forwarding node of node $s_{i}$, then $x\left(s_{i}, s_{j}\right)=1$, otherwise $x\left(s_{i}, s_{j}\right)=0 . e\left(s_{i}\right.$, $s_{j}$ ) represents the energy consumption on the edge from node $s_{i}$ to $s_{j}$ consists of three components: node $s_{i}$ transmitting data, node $s_{i}$ receiving data and fusing data. In Equation 2, the constraint specifies that the node $s_{i}$ has only one forwarding node.

\section{Energy efficient data fusion during data collection}

In this section, we will discuss the performance of data fusion in cluster hierarchy and make a priority analysis on energy consumption of network, where the cost of transmitting and receiving data are also both considered.

\section{A. Network topology}

As shown in Figure 2, we divide the network into cluster and each cluster is composed by a series of grids. Both cluster and grid are square structure. There are 


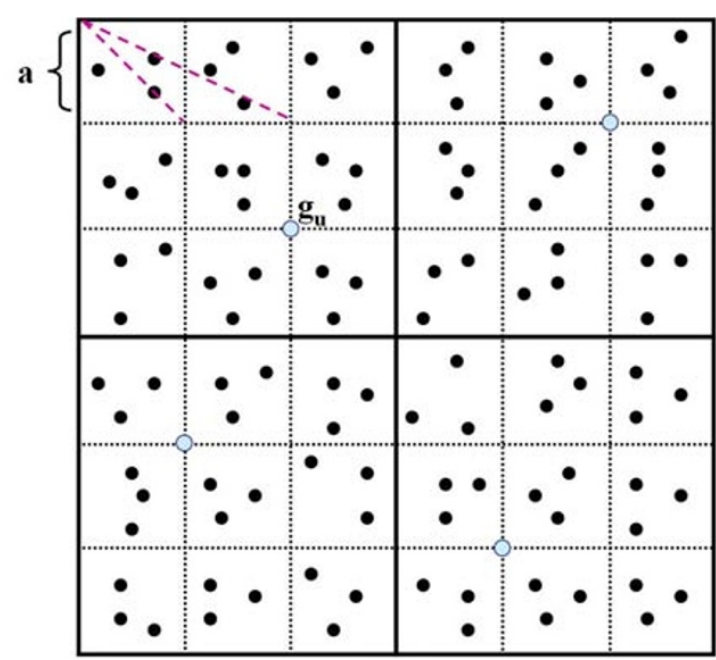

Figure 2 An example of network division.

many sensor nodes randomly deployed in each cluster, and the blue dots are the mobile node, which acted as cluster head. With increasing the communication distance, the energy consumption increases quickly, so it should avoid a long distance of single hop. Therefore, we adopt three kinds of communication distance for such topology. The longest one is used for mobile agent node to receive and transmit data from a long distance between different cluster, marked as $R$. The rest two short transmission distances are, respectively, used for data transmission between different sensor nodes in same and neighbor grid, marked as $r$ and $r$ '. The communication range should also cover all the possible nodes to guarantee that there is no data loss during the transmission. Suppose the length of each grid is $a$, this condition for any two sensor nodes in the same grid that can communicate is $r \geq \sqrt{2} a$ while any two sensor nodes in the neighbor grid is $r^{\prime}=\sqrt{5} a$.

The square grid that we adopted has strong regularity and is easy to be analyzed. When the sensor nodes are deployed in uniform, the number of sensor node in each grid is same. Without loss of generality, we suppose that all nodes are random deployed, which make the number of sensor nodes in each cluster and grid are different. Then, we will analyze the energy efficiency of intra-grid and inter-grid.

\section{B. Energy efficient data fusion of intra-grid}

This section focuses on analysis the energy efficiency of intra-grid data fusion when sensor nodes are random deployed. For a mobile agent node $g_{u} \in G$, its location is regarded as the reference point of its cluster, denoted as $O\left(g_{u}\right)$. The grids are numbered according to relative position to $g_{u}$. For example, the number in the cluster of $g_{u}$ in Figure 2 can be expressed as

$$
\left[\begin{array}{ccc}
(-2,2) & (-1,2) & (1,2) \\
(-2,1) & (-1,1) & (1,1) \\
(-2,-1) & (-1,-1) & (1,-1)
\end{array}\right] .
$$

We use $C_{u}$ represent the cluster that mobile agent node $g_{u}$ belongs to and $g_{u}(x, y)$ represent the grid with coordinate $(x, y)$. Let $E_{u}(x, y)$ represent the total energy consumption of all sensor nodes in grid $g_{u}(x, y)$ for data collection during time $T$, according to energy model given in Section 3,

$$
E_{u}(x, y)=D_{u t}(x, y) e_{t}+D_{u r}(x, y) e_{r}+D_{u f}(x, y) e_{f},
$$

where $D_{u t}(x, y), D_{u r}(x, y), D_{u f}(x, y)$ represent the total data amount of sensor nodes of grid $g_{u}(x, y)$ in time $T$ for transmitting, receiving, and fusing.

From Equation 4, it can be seen that the energy consumption is determined by the data amount of the above three operations. Aiming for energy efficiency, we need to judge whether sensor nodes should complete data fusion process during intra-grid data collection. $D$ $\left(s_{i}\right)$ and $D\left(s_{j}\right)$ represent the data amount generated by node $s_{i}$ and $s_{j}$ in grid $g_{u}(x, y)$. The decision of whether proceeding data fusion of intra-grid is based on the following theorem.

Theorem 1: $\forall s_{i}, s_{j} \in g_{u}(x, y)$, the condition of intra-grid data fusion that can save energy is: $\sigma>\frac{D\left(s_{i}\right)\left[e_{t}(a)+e_{r}+e_{f}\right]+D\left(s_{j}\right) e_{f}}{\min \left(D\left(s_{i}\right), D\left(s_{j}\right)\right) e_{t}(\sqrt{2} a)}$.

Proof: There are two situations, one is that there is no data fusion process of intra-grid and all the sensory data are directly sent to the neighbor grid. Then, the total energy consumption of node $s_{i}$ and $s_{j}$ are only caused by transmission to the neighbor grid and can be calculated as

$$
E_{1}=\left[D\left(s_{i}\right)+D\left(s_{j}\right)\right] e_{t}(\sqrt{2} a) .
$$

The other situation is to execute inter-grid data fusion, where the node $s_{i}$ first sends the data to node $s_{j}$. After fusion process, $s_{j}$ sends the fused data to neighbor grid. The total energy consumption of node $s_{i}$ and $s_{j}$ can be calculated as

$$
\begin{aligned}
E_{2}= & D\left(s_{i}\right) e_{t}(a)+D\left(s_{i}\right) e_{r}+\left[D\left(s_{i}\right)+D\left(s_{j}\right)\right] e_{f} \\
& +\left[\max \left(D\left(s_{i}\right), D\left(s_{j}\right)\right)\right. \\
& \left.+\min \left(D\left(s_{i}\right), D\left(s_{j}\right)\right)(1-\sigma)\right] e_{t}(\sqrt{2} a) .
\end{aligned}
$$

It can be deduced that the condition of intra-grid data fusion can save energy is

$$
\begin{aligned}
E_{2}-E_{1}= & D\left(s_{i}\right) e_{t}(a)+D\left(s_{i}\right) e_{r}+\left[D\left(s_{i}\right)+D\left(s_{j}\right)\right] e_{f} \\
& -\min \left(D\left(s_{i}\right), D\left(s_{j}\right)\right) \sigma e_{t}(\sqrt{2} a)<0 \\
\Rightarrow & \sigma>\frac{D\left(s_{i}\right)\left[e_{t}(a)+e_{r}+e_{f}\right]+D\left(s_{j}\right) e_{f}}{\min \left(D\left(s_{i}\right), D\left(s_{j}\right)\right) e_{t}(\sqrt{2} a)} .
\end{aligned}
$$


Hence, Theorem 1 is proved.

It can be seen from Theorem 1 that the energy-saving degree of fusion process is determined by the relativity of the processed data when the data amount is known.

\section{Energy efficient data fusion of inter-grid}

In Section 4.2, we have proved the condition of intragrid data fusion that can save energy. Now, we will analyze the performance of data fusion of inter-grid. Based on the above analysis, this problem can be transformed to judge whether fusing the data from two different grids can save energy. As shown in Figure 3, $g_{u}(x, y)$ and $g_{u}\left(x^{\prime}, y^{\prime}\right)$ are two grids which have sensory data need to transmit to the mobile agent node $g_{u}$. To complete data collection, the nodes in two grids need to establish the path that can arrive at $g_{u}$. If the data in two grids need to be fused, their data transmission routes ought to meet at one grid before reaching the destination $g_{u}$. For example, the route of $s_{i}$ in $g_{u}(x, y)$ and $s_{j}$ in $g_{u}\left(x^{\prime}, y^{\prime}\right)$ meet at grid $g_{u}\left(x_{0}, y_{0}\right)$.

Theorem 2: $\forall s_{i} \in g_{u}(x, y), s_{j} \in g_{u}\left(x^{\prime}, y^{\prime}\right)$, the condition of inter-grid data fusion that can save energy is $\sigma>\frac{\left[D\left(s_{i}\right)+D\left(s_{j}\right)\right] e_{f}}{\min \left(D\left(s_{i}\right), D\left(s_{j}\right)\right)\left(e_{t}(\sqrt{2} a)+e_{r}\right)\left(\left|x_{0}\right|+\left|y_{0}\right|\right)}$

Proof: There are two situations. One is that the data of the two nodes are separately transmitted to the mobile agent node without any cross. The total energy consumption of $s_{i}$ and $s_{j}$ can be calculated as

$$
\begin{aligned}
E_{1}= & D\left(s_{i}\right) e_{t} N_{t}\left(s_{i}\right)+D\left(s_{i}\right) e_{r} N_{r}\left(s_{r}\right) N_{t}\left(s_{j}\right) \\
& +D\left(s_{j}\right) e_{r} N_{r}\left(s_{j}\right)
\end{aligned}
$$

where $N_{t}\left(s_{i}\right)$ and $N_{t}\left(s_{j}\right)$ represent the number of transmission for data generated by $s_{i}$ and $s_{j}$ before they arrived at grid $g_{u}$, and $N_{r}\left(s_{i}\right)$ and $N_{r}\left(s_{j}\right)$ represent the

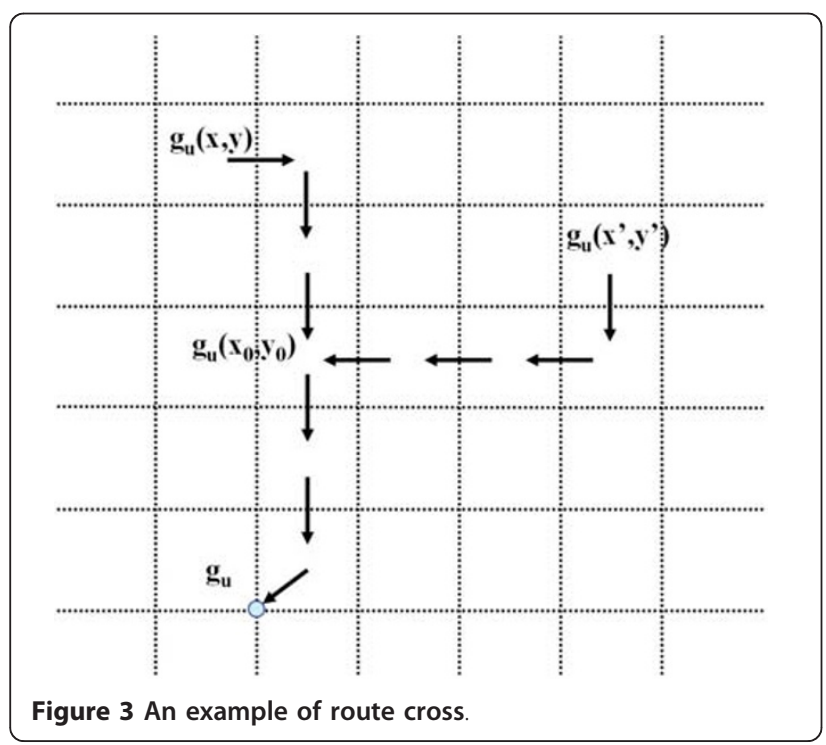

number of reception for data generated by $s_{i}$ and $s_{j}$, all these parameters can be obtained by the coordinates referred to the grids.

$$
\left\{\begin{array}{c}
N_{t}\left(s_{i}\right)=|x|+|y|, \\
N_{r}\left(s_{i}\right)=|x|+|y|-1, \\
N_{t}\left(s_{j}\right)=\left|x^{\prime}\right|+\left|y^{\prime}\right|, \\
N_{r}\left(s_{j}\right)=\left|x^{\prime}\right|+\left|y^{\prime}\right|-1 .
\end{array}\right.
$$

As mobile agent nodes are not limited by energy, the energy consumption of receiving data by mobile agent node $g_{u}$ is not considered, and also not counted in $N_{r}\left(s_{i}\right)$ and $N_{r}\left(s_{j}\right)$ of Equation 9. Substitute it into Equation 8:

$$
\begin{aligned}
E_{1}= & D\left(s_{i}\right) e_{t}(\sqrt{2} a)(|x|+|y|) \\
& +D\left(s_{i}\right) e_{r}(\sqrt{2} a)(|x|+|y|-1) \\
= & D\left(s_{i}\right)\left[e_{t}(\sqrt{2} a)(|x|+|y|)+e_{r}(|x|+|y|-1)\right] \\
= & D\left(s_{j}\right)\left[e_{t}(\sqrt{2} a)\left(\left|x^{\prime}\right|+\left|y^{\prime}\right|\right)\right. \\
& \left.+e_{r}\left(\left|x^{\prime}\right|+\left|y^{\prime}\right|-1\right)\right] .
\end{aligned}
$$

In the other situation, the routes of $s_{i}$ and $s_{j}$ to mobile agent node $g_{u}$ meet at grid $g_{u}\left(x_{0}, y_{0}\right)$, then their data are fused.

The total energy consumption can be calculated as

$$
\begin{aligned}
E_{2}= & D\left(s_{i}\right)\left[e_{t} N_{t 1}\left(s_{i}\right)+e_{r} N_{r 1}\left(s_{i}\right)\right]+D\left(s_{j}\right)\left[e_{t} N_{t 1}\left(s_{j}\right)\right. \\
& \left.+e_{r} N_{r 1}\left(s_{j}\right)\right]+\left[D\left(s_{i}\right)+D\left(s_{j}\right)\right] e_{f} \\
& +\left[\max \left(D\left(s_{i}\right), D\left(s_{j}\right)\right)\right. \\
& \left.+(1-\sigma) \min \left(D\left(s_{i}\right), D\left(s_{j}\right)\right)\right]\left[N_{t 2}\left(s_{i}, s_{j}\right) e_{t}\right. \\
& \left.+N_{r 2}\left(s_{i}, s_{j}\right) e_{r}\right]
\end{aligned}
$$

where $N_{t 1}\left(s_{i}\right)$ and $N_{t 1}\left(s_{j}\right)$ represent the number of transmission for data generated by $s_{i}$ and $s_{j}$ before arriving at $g_{u}\left(x_{0}, y_{0}\right) . N_{r 1}\left(s_{i}\right)$ and $N_{r 1}\left(s_{j}\right)$ represent the number of receiving for data generated by $s_{i}$ and $s_{j}$ before arriving at $g_{u}\left(x_{0}, y_{0}\right) . N_{t 2}\left(s_{i}\right)$ and $N_{r 2}\left(s_{j}\right)$ represent the number of transmission and receiving for data fused by $s_{i}$ and $s_{j}$.

$$
\left\{\begin{array}{c}
N_{t 1}\left(s_{i}\right)=\left|x-x_{0}\right|+\left|y-y_{0}\right|, \\
N_{r 1}\left(s_{i}\right)=\left|x-x_{0}\right|+\left|y-y_{0}\right|-1, \\
N_{t 1}\left(s_{j}\right)=\left|x^{\prime}-x_{0}\right|+\left|y^{\prime}-y_{0}\right|, \\
N_{r 1}\left(s_{j}\right)=\left|x^{\prime}-x_{0}\right|+\left|y^{\prime}-y_{0}\right|-1, \\
N_{t 2}\left(s_{i}, s_{j}\right)=\left|x_{0}\right|+\left|y_{0}\right|, \\
N_{r 2}\left(s_{i}, s_{j}\right)=\left|x_{0}\right|+\left|y_{0}\right|-1 .
\end{array}\right.
$$

Substitute to Equation 10:

$$
\begin{aligned}
E_{2}= & D\left(s_{i}\right)\left[\left(e_{t}(\sqrt{2} a)+e_{r}\right)\left(\left|x-x_{0}\right|+\left|y-y_{0}\right|\right)-e_{r}+e_{f}\right] \\
& +D\left(s_{j}\right)\left[\left(e_{t}(\sqrt{2} a)+e_{r}\right)\left(\left|x^{\prime}-x_{0}\right|+\left|y^{\prime}-y_{0}\right|\right)-e_{r}+e_{f}\right] \\
& +\left[\max \left(D\left(s_{i}\right), D\left(s_{j}\right)\right)+(1-\sigma) \min \left(D\left(s_{i}\right), D\left(s_{j}\right)\right)\right] \\
& \cdot\left(e_{t}(\sqrt{2} a)+e_{r}\right)\left(\left|x_{0}\right|+\left|y_{0}\right|\right) .
\end{aligned}
$$

The condition of inter-grid data fusion that can save energy is 


$$
\begin{aligned}
E_{2}- & E_{1}=D\left(s_{i}\right)\left[( e _ { t } ( \sqrt { 2 } a ) + e _ { r } ) \left(\left|x-x_{0}\right|+\left|y-y_{0}\right|\right.\right. \\
& \left.\left.-|x|-|y|+e_{f}\right)\right]+D\left(s_{j}\right)\left[( e _ { t } ( \sqrt { 2 } a ) + e _ { r } ) \left(\left|x^{\prime}-x_{0}\right|\right.\right. \\
& \left.\left.+\left|y^{\prime}-y_{0}\right|-\left|x^{\prime}\right|-\left|y^{\prime}\right|\right)+e_{f}\right] \\
& +\left[\max \left(D\left(s_{i}\right), D\left(s_{j}\right)\right)+(1-\sigma) \min \left(D\left(s_{i}\right), D\left(s_{j}\right)\right)\right] \\
& +\left(e_{t}(\sqrt{2} a)+e_{r}\right)\left(\left|x_{0}\right|+\left|y_{0}\right|\right)<0 .
\end{aligned}
$$

As $|x| \geq\left|x_{0}\right|,\left|x^{\prime}\right| \geq\left|x_{0}\right|$, the former can be simplified:

$$
\begin{aligned}
E_{2}-E_{1}= & {\left[D\left(s_{i}\right)+D\left(s_{j}\right)\right] e_{f}-\left(e_{t}(\sqrt{2} a)\right.} \\
& \left.+e_{r}\right)\left(\left|x_{0}\right|+\left|\gamma_{0}\right|\right) \sigma \min \left(D\left(s_{i}\right), D\left(s_{j}\right)\right)<0 \\
\Rightarrow & \sigma>\frac{\left[D\left(s_{i}\right)+D\left(s_{j}\right)\right] e_{f}}{\min \left(D\left(s_{i}\right), D\left(s_{j}\right)\right)\left(e_{t}(\sqrt{2} a)+e_{r}\right)\left(\left|x_{0}\right|+\left|\gamma_{0}\right|\right)} .
\end{aligned}
$$

Hence, Theorem 2 is proved.

We can use Theorem 2 to direct the routing establishment and find the optimization strategy for saving energy. According to this point, the nodes in the same grid may establish different routes to the mobile agent node.

\section{Energy efficient fusion-driven routing with mobile agent}

In this section, we design an EEFR based on the above analysis. Using the mobility of agent node, our proposed fusion-driven routing aims to attain the energy efficient data collection with random node deployment.

\section{A. Cluster and grid division}

The topology of EEFR is an extension based on the classical hierarchy GAP, which is also to divide the network into a series of square grids. In the network, we deployed mobile agents in uniform. The ordinary sensor nodes form the cluster structure around these mobile agent nodes. Similar to the sink node, the mobile agent nodes are not limited by energy supply, so they act as the cluster head and are responsible for the data collection management of this cluster. As the cluster head, the mobile agent node should have the ability of managing all the sensor nodes in the cluster simultaneously. Different from other cluster routing, the cluster head in EEFR does not need to be selected by rotation method from ordinary sensor nodes. To reduce the cost of network, the number of mobile agent nodes should be as less as possible which means the area managed by mobile agent nodes should be as large as possible. To guarantee the communication of two mobile agent nodes in the neighbor cluster, the number of mobile agent nodes is limited by their communication ability. Let $R$ represent the communication distance of mobile agent nodes, the maximum area of cluster is

$$
S_{c}=\frac{R^{2}}{5}
$$

If the cover area of whole multimedia sensor network is $S$, the minimum amount of needed mobile agent can be calculated as

$$
N_{a}=\frac{S}{S_{c}}=\frac{5 S}{R^{2}} .
$$

The topology division of network includes the following two steps. The first is to divide the network into a series of larger equal square area and each square represents one cluster. There is only one mobile agent node in each cluster which can move freely. The second one is to further divide the cluster into many smaller equal square, and each square represents one grid. For the sake of avoiding data loss during transmission, the size of grid is not too large but should guarantee the communication of two random nodes in the neighbor grids. When the sensor node knows which grid it belongs to, it needs to broadcast the information with identification and receive the information from other nodes in the neighbor grid.

For completing more complicated tasks, sensor nodes are equipped with many different sensors so that the traditional data collection model is not valid. The network needs to complete more than one task, that one task is respond to one data set as described in Definition 3.1, then all the data sets compose of the data space. The mobile agent node needs to arrange the suitable nodes to complete data collection according to different tasks.

As shown in Figure 4, the operation procedure of EEFR is divided into a number of rounds and each round includes two phases, namely set-up phase and steady-state phase. The working process of set-up phase needs to complete the movement of mobile agent node, the establishment of routes, and the distribution of

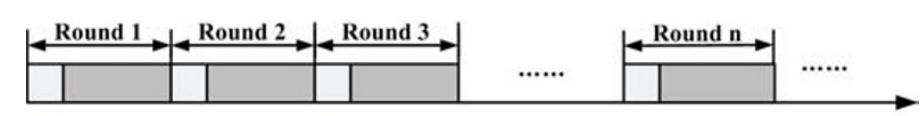

Set-up phase 
time-slot allocation. In steady-state phase, the sensor nodes complete the data collection under the management of mobile agent node.

\section{B. Movement of mobile agent node}

When the mobile agent node moves to a new position, the coordinate of the grid will also change. Only the sensor nodes in the grids with the coordinate of $(1,1)$, $(1,-1),(-1,-1)$, and $(-1,1)$ can directly send data to mobile agent node while all the other sensor nodes need to send their data to the mobile agent node by multihop method. In EEFR, multi-hop routes are formed between neighbor grids from source node to the mobile agent node, which can guarantee the sensory data generated by the farthest node not be lost. The data collection of inter-cluster starts from the outermost grid and ends when all data are transmitted to the mobile agent node. One-to-one or many-to-one mappings are formed among sensor nodes of neighbor grid. If a sensor node in grid $g_{u}(x, y)$ has sensory data to be transmitted, it needs to select the corresponding relay node from its neighbor grids which is much closer to the mobile agent node. For example, when $x, y>0$, the selected relay node should be in the grid $g_{u}(x-1, y)$ or $g_{u}(x, y-1)$. In this case, $g_{u}(x, y)$ acts as a source grid, while $g_{u}(x-1, y)$ or $g_{u}(x, y-1)$ acts as the destination grid.

The data collection involves in many kinds of sensors to complete different tasks in EEFR. According to the Definition 1 in Section 3, the data collection space $\widehat{D}$ consists of $k$ data sets and denoted as $\widehat{D}=D_{1} \times D_{2} \times \cdots \times D_{k}$. Each data set includes sensory data in different attributes.

The sink node should confirm the data set corresponding to each task and the data collection space composed by all data sets, then send these information to each mobile agent node. As the management node of cluster, the mobile agent node needs to judge which specific nodes will be used to complete the different tasks. To realize the efficient data collection, the effect of data fusion on energy consumption should be fully considered. The effective data fusion can reduce the energy consumption, while the reduced data amount is determined by the data coefficient relativity. The current research shows that the data coefficient relativity is affected by the distance among nodes. Although there are other kinds of models, the common result shows that the data coefficient relativity among nodes reduces as the increasing the space distance. Here, we adopt a model proposed by [5]

$$
\rho=\left(1-d / d_{s}\right) \cdot f \cdot p,
$$

where $d$ and $d_{s}$ represent the maximum correlation distance between nodes and the space distance between two sensor nodes, $f$ represents the effect of the fusion algorithm on the data correlations, and $p$ is the data attribute.

The mobile agent node is responsible to find the efficient data fusion which can reduce the energy consumption in its cluster and achieve the optimization target to direct the establishment of routing. Based on Theorems 1 and 2, we can judge whether the data fusion is effective and take use of the mobility of agent node to maximum the performance of saving energy by data fusion. $s_{i}$ and $s_{j}$ are any two nodes in different grid of the same cluster. The following Lemma 1 gives the calculation method of finding the mobile agent position which can realize the efficient data collection using the efficient data fusion in maximum.

Lemma 1: The energy efficiency can be realized if the location of a mobile agent node can meet the requirement of

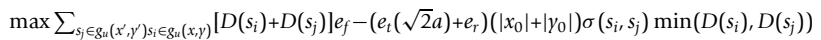

Proof: If data fusion is not adopted, for any sensor node $s_{i} \in g_{u}(x, y)$, the energy consumption of collecting the data generated by $s_{i}$ can be calculated as

$$
\begin{aligned}
E_{1}\left(s_{i}\right)= & D\left(s_{i}\right) e_{t}(\sqrt{2} a)(|x|+|y|) \\
& +D\left(s_{i}\right) e_{r}(|x|+|y|-1) .
\end{aligned}
$$

This moment, the minimum total energy consumption of the cluster $g_{u}$ is

$$
\begin{aligned}
E_{u 1}= & \sum_{s_{i} \in C_{u}} E_{1}\left(s_{i}\right) \\
= & \sum_{s_{i} \in C_{u}}\left[D\left(s_{i}\right) e_{t}(\sqrt{2} a)(|x|+|\gamma|)\right. \\
& +D\left(s_{i}\right) e_{r}(|x|+|y|-1) .
\end{aligned}
$$

If the data fusion is adopted, both of intra-grid and inter-grid fusion processes are possible to reduce energy consumption. Here, the minimum energy consumption becomes

$$
E_{u 2}=E_{u 1}-E_{\text {intra-f }}-E_{\text {inter-f, }}
$$

where $E_{\text {intra-f }}$ and $E_{\text {inter-f }}$ represent the saved the energy consumption by data fusion of intra-grid and inter-grid. The fusion process of intra-grid is not affected by the location of mobile agent node. It is only necessary to consider how to maximize the saving energy efficient of inter-grid fusion process, which can be described as

$$
\begin{aligned}
\max \left(E_{\text {inter-f }}\right)= & \max \sum_{s_{j} \in g_{u}\left(x^{\prime}, y^{\prime}\right)} \sum_{s_{i} \in g_{u}(x, y)}\left[D\left(s_{i}\right)\right. \\
& \left.+D\left(s_{j}\right)\right] e_{f} \\
& -\left(e_{t}(\sqrt{2} a)+e_{r}\right)\left(\left|x_{0}\right|\right. \\
& \left.+\left|y_{0}\right|\right) \sigma\left(s_{i}, s_{j}\right) \\
& \cdot \min \left(D\left(s_{i}\right), D\left(s_{j}\right)\right) .
\end{aligned}
$$


Hence, Lemma 1 is proved.

According to different tasks, the mobile agent node can find the optimized position by Lemma 1, then complete the establishment of intra-cluster routing.

\section{Routing establishment}

Although the minimizing energy consumption is the prior target during the routing establishment, EEFR also try to balance the energy consumption among different sensor nodes. As the sensor nodes are deployed in high density, more than one node might meet the requirement of minimizing energy consumption during routing establishment. For the sake of balancing the energy consumption, the node with more remaining energy should be selected to undertake the routing task. The routing selection includes three steps.

The first step: the mobile agent node determines the sensor nodes to complete data collection in its cluster according to different tasks, then calculates the optimization position by Lemma 1. As shown in Figure 5a, the three optimization positions of mobile agent nodes data are separately found according to different data sets $D_{1}$, $D_{2}$, and $D_{3}$ generated by different tasks. Where, the black, purple, and green dots are the nodes generating data sets $D_{1}, D_{2}$, and $D_{3}$.

The second step: the mobile agent node determines the sensor nodes that participate the intra-grid data fusion by Theorem 1, and point the nodes with more remaining energy to complete the fusion by Theorem 1 . As shown in Figure 5b, the nodes with short distance in the same grid participate intra-grid fusion process since they can meet the requirement of Theorem 1, while the nodes in long distance are forbidden in such operations.

The third step: the routing selection of inter-grid is completed by the mobile agent node according to Theorem 2, which needs to maximize the performance of saving energy by inter-grid data fusion. As shown in Figure $5 \mathrm{c}$, the nodes with more remaining energy that can meet the requirement of saving energy are chosen during routing establishment. The coordinate $\left(x_{0}, y_{0}\right)$ of route intersection from node $s_{i}$ in grid $g_{u}(x, y)$ and $s_{j}$ in grid $g_{u}\left(x^{\prime}, y^{\prime}\right)$ to mobile agent $g\left(x_{0}, y_{0}\right)$ in a grid should satisfy the following condition:

$$
\left\{\begin{aligned}
\left|x_{0}\right|+\left|y_{0}\right|>\frac{\left[D\left(s_{i}\right)+D\left(s_{j}\right)\right] e_{f}}{\min (D(s i), D(s j))\left(e_{t}(\sqrt{2} a)+e_{r}\right) \sigma} \\
\left|x_{0}\right| \leq \max \left(|x|,\left|x^{\prime}\right|\right) \\
\left|y_{0}\right| \leq \max \left(|y|,\left|y^{\prime}\right|\right)
\end{aligned}\right.
$$

For saving energy consumption, it is a general method to make the sensor nodes turn to sleep when they do not have any task. EEFR routing also adopts time-slot allocation to reduce the probability of communication collision and save energy. Let $t_{\text {intra }}$ and $t_{\text {inter }}$ represent

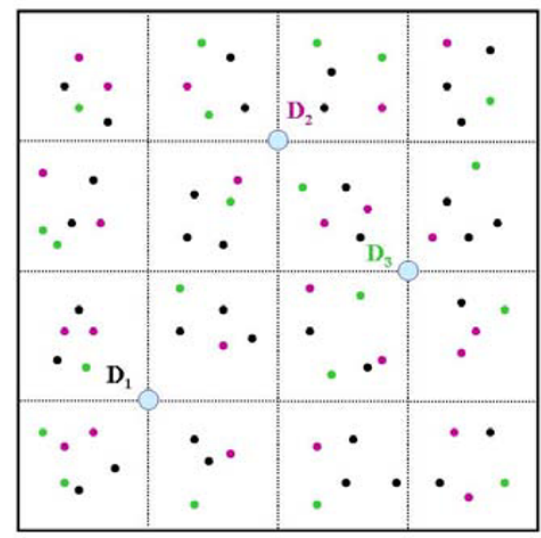

(a) Locating of mobile agent

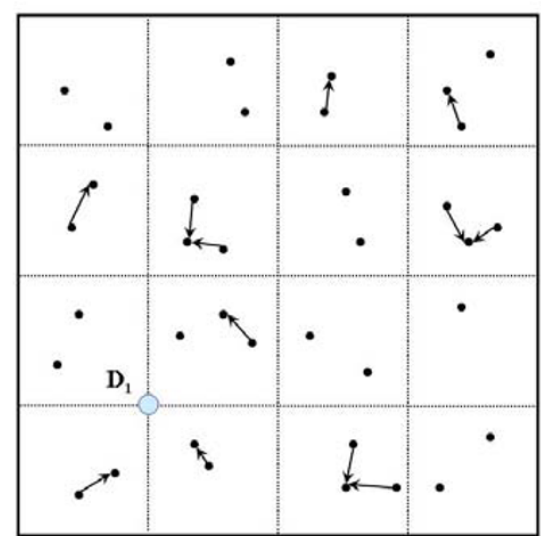

(b) Confirmation of intra-fused node

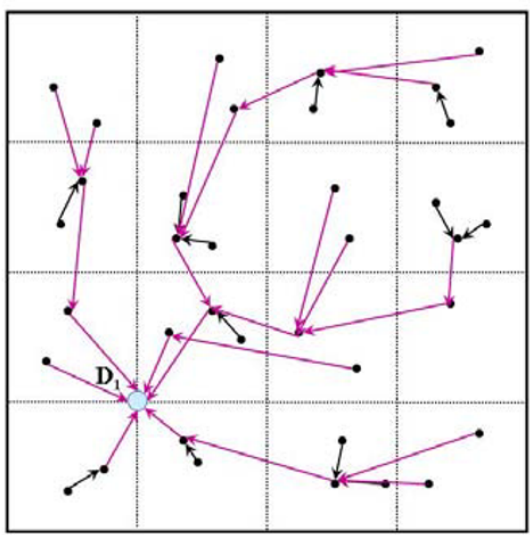

(c) Routes establishment of inter-grid

Figure 5 Working process of EEFR routing

the consumed time of intra-grid and inter-grid data fusion process. $n_{\max -f}$ represents the maximum number of each grid to complete intra-grid fusion process. $n_{t}(x$, $y)$ is the number of sending data from grid $g_{u}(x, y)$ to 
other grids, $n_{r}(x, y)$ is the number of receiving data of grid $g_{u}(x, y)$ from other grids. $T_{u}$ is the running time of a round. The following Lemma 2 gives the allocated operating time of a random grid $g_{u}(x, y)$ :

Lemma 2: For $\forall g_{u}(x, y)$, the allocated operating time is $\left\{n_{\max -f} t_{\text {intra }}+\left[n_{t}(x, y)+n_{r}(x, y)\right] t_{\text {inter }}\right\} T_{u}$

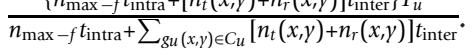

Proof: According to the data collection process, it is firstly to complete the data transfer and fusion of intragrid by distributed method. Hence, each grid is allocated with the same operating time for intra-grid, which can be calculated as

$$
T_{\text {intra }}=t_{\text {intra }} \cdot n_{\max -f} .
$$

Then, the time of data transfer and fusion process between grids can be calculated as

$$
T_{\text {inter }}=\sum_{s_{u}(x, y) \in C_{u}}\left[n_{t}(x, y)+n_{r}(x, y)\right] t_{\text {inter }} .
$$

Hence, the allocated operation time of each grid is

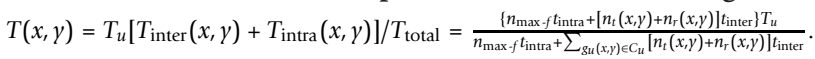

Hence, Lemma 2 is proved.

Lemma 2 shows that the allocated time of grid is determined by the task amount undertaken by its nodes, where the longer time will be allocated because of more tasks.

\section{Simulation and result analysis}

In this section, we evaluate the performance of the proposed EEFR routing by experiments. Our experiments demonstrate the energy consumption of network and node survival condition by adopting EEFR with different parameters. We further evaluate the performance of EEFR by comparing with a clustering hierarchy based on cellular topology named CHCT [19] and LEACHMT which modified based on classic LEACH algorithm [13].

\section{A. Simulation environment}

In our simulations, 200 sensor nodes are randomly deployed in a square area of $300 \times 300 \mathrm{~m}^{2}$. There are some homogenous mobile agent nodes uniformly distributed in the square area and only one sink node locates at the center. The sink node and all mobile agent nodes are not limited by energy supply, while the sensor nodes are all stationary and have the same initial energy $20 \mathrm{~J}$. Each round lasts for $300 \mathrm{~s}$. For the radio model, the parameters are set as follows: $E_{\text {elec }}=50 \mathrm{~nJ} /$ bit, $\varepsilon_{\mathrm{amp}}=0.0013 \mathrm{pJ} / \mathrm{bit} / \mathrm{m}^{4}$. All simulations are based on a collision-free MAC protocol without data loss. The sensory data generated by each sensor node is 500 bit. In order to facilitate read, the related system parameters are listed in Table 1.
We use network lifetime and the number of alive nodes to evaluate the performance in our simulations. Generally, the network lifetime can be measured by three methods. One is the time when the first node exhausts its energy, the second is the time when the dead nodes reach a certain degree, and third is the time of all nodes dead. Here, we choose the third one as the network lifetime. The number of alive sensor node means the node still can normally work, which decreases with the network operation.

\section{B. Network lifetime and node survival condition}

In this simulation, we focus on evaluating the performance of EEFR by network lifetime and remaining energy. The network lifetime is recorded as the time when all the sensor nodes are energy exhausted. The longer network lifetime means the higher efficiency of saving energy. When the first dead node appears, the less remaining energy of other nodes means the better energy balancing performance.

The effect of network structure on EEFR performance is also considered by changing the number of the agent vary from 5 to 8 . The coefficient relativity of data in neighbor grids is fixed at 0.3 . Figure 6 gives the situation of energy consumption of whole network with time increasing. When the energy consumption of network reaches $40000 \mathrm{~J}$, the whole energy of network are thoroughly consumed and will not change but keep constant. Then, the whole operating time is the network lifetime. We also notice that the network lifetime is longer with increasing the agent amount. This is because the cluster area is decreased by the increasing number of mobile agent nodes, which results in the decreasing of hop number. Therefore, the energy consumption of retransmission is saved.

Figure 7 shows the statistic result of the changing about the number of alive nodes along with network operating. We observe that the appearance time of the first and last dead node is similar, which means the energy consumption of network adopted by EEFR is quite balanced. It also can be seen that the increasing of mobile agent nodes delays the appearance time of dead node. The increasing number of mobile agent reduced the cluster area, then the hop number of the nodes in the far distance from the mobile agent node correspondingly reduced. As a result, the energy consumption difference between the node with largest and smallest hop number obviously decreased.

Figure 8 illustrates the network lifetime adopted by EEFR with different data correlation coefficient. We suppose the correlation coefficient of the sensory data in neighbor grids is changed from 0.1 to 0.8 , and the correlation coefficient of sensory data reduces 0.1 when the interval of grid increases 1 . It can be seen that the 


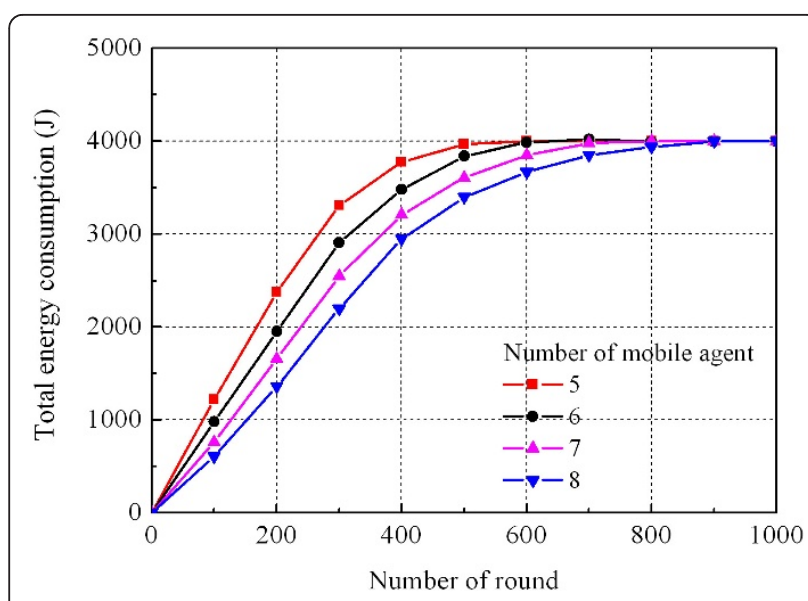

Figure 6 Energy consumption of network.

network lifetime increases with increasing data correlation coefficient.

\section{Comparison with other routings}

In this simulation, we evaluate the performance of EEFR by comparing it with a clustering hierarchy based on cellular topology (CHCT) and LEACH-MT. CHCT adopts the conception of virtual grid to form the cellular clusters in WMSNs and considers the remaining energy of sensor nodes during routing establishment. Five mobile agent nodes are used in the experiment operated by EEFR. LEACH-MT is modified from LEACH to make it be able to operate under a large-scale deployment. The multi-hop mechanism is introduced in LEACH-MT for data transmission among clusters. The rest of LEACH-MT is as same as LEACH. Fusion process is employed during data collection in these three protocols. Figure 9 illustrates the change of their energy consumption with correlation coefficient at 0.3 . It can be seen that the energy consumption of network

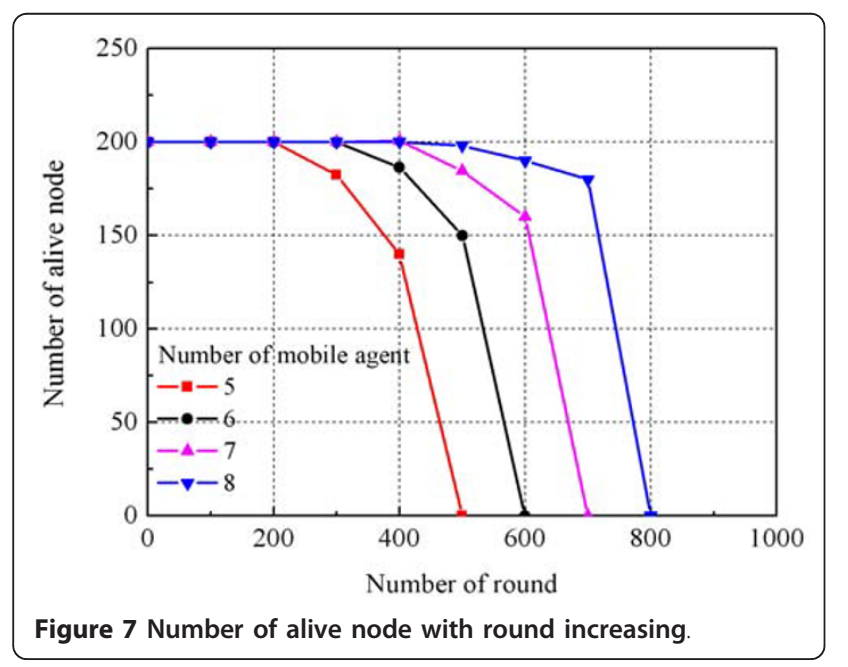

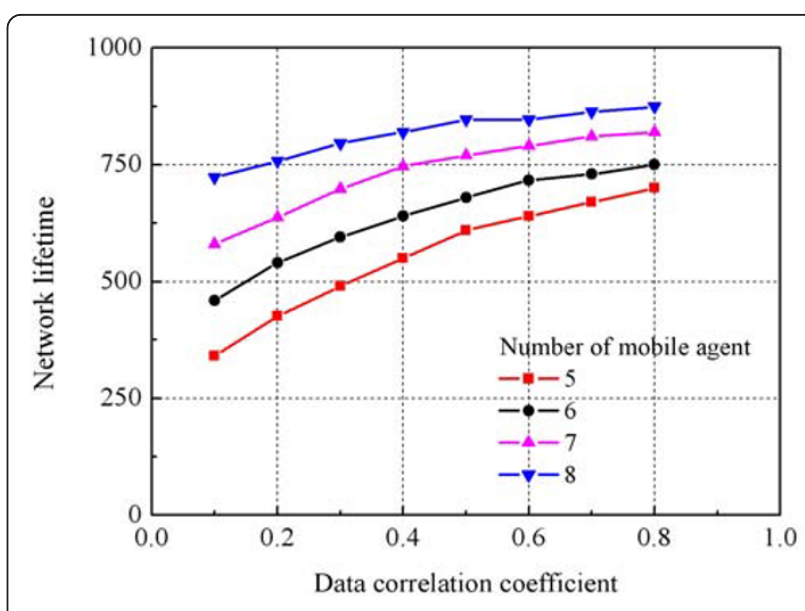

Figure 8 Comparison of network lifetime under different data coefficient relativity.

adopted by EEFR is always lower than that of CHCT and LEACH-MT with the same data correlation coefficient.

\section{Conclusion}

For the energy limited WMSNs, the most challenge problem is how to effectively use the energy of network during data collection. In this article, we theoretically analyze the energy consumption of sensor nodes during data collection when the nodes are random deployed. We find that fusion process can reduce the energy consumption, and the efficiency of saving energy is determined by data correlation coefficient. Then, we study the mobility of mobile agent node on maximizing the saving energy efficiency of data fusion. Finally, we design an EEFR based on cluster hierarchy for WMSNs, where the cluster structure is formed based on square grid topology. Extensive simulations are performed to validate our proposed EEFR. Simulation results show that

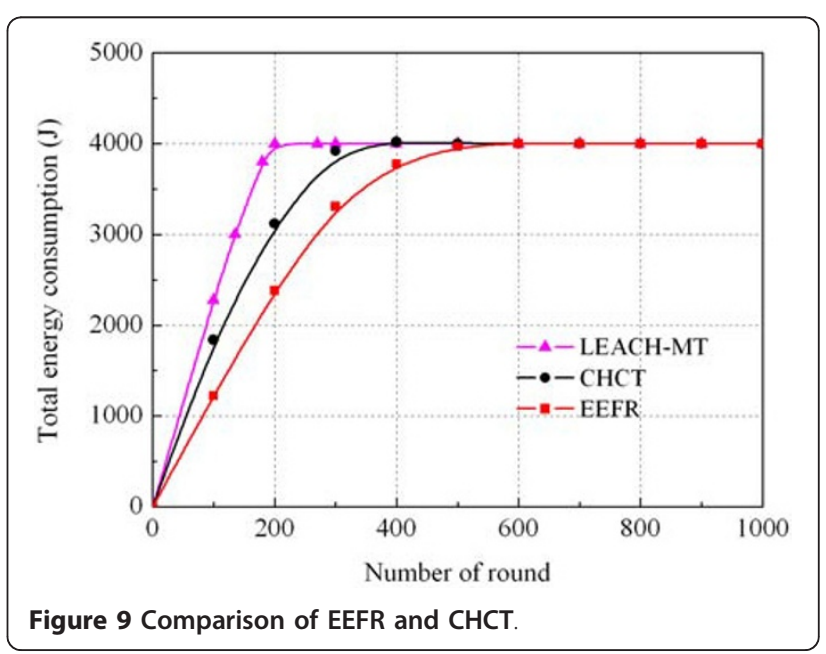


EEFR shows high performance in decreasing energy consumption with node random deployment.

\section{Acknowledgements}

This study was partially supported by the National Science Foundation of China (NSFC) under Grant No. 61103234.

\section{Author details}

${ }^{1}$ School of Computer Science and Engineering, Dalian University of Technology, Dalian, Liaoning, China ${ }^{2}$ School of Computer Science and Engineering, Seoul National University, Seoul, Korea

\section{Competing interests}

The authors declare that they have no competing interests.

Received: 28 June 2011 Accepted: 27 October 2011

Published: 27 October 2011

\section{References}

1. IF Akyildiz, T Melodia, KR Chowdhury, A survey on wireless multimedia sensor networks. Comput Networks. 51, $921-960$ (2007). doi:10.1016/j. comnet.2006.10.002

2. M Chen, M Guizani, M Jo, Mobile multimedia sensor networks: architecture and routing. MobiWorld 2011. in Conjunction with the 30th IEEE International Conference on Computer Communications (Infocom 2011), Shanghai, China, 10-15 (April 2011)

3. E Gurses, OB Akan, Multimedia communication in wireless sensor networks. Ann Telecommun. 60(7-8), 799-827 (2005)

4. S Misra, M Reisslein, G Xue, A survey on multimedia streaming in wireless sensor networks. IEEE Commun Surv Tutorials 10(4), 18-39 (2008)

5. K Lin, L Wang, K Li, L Shu, Multi-attribute data fusion for energy equilibrium routing in wireless sensor networks. KSII Trans Internet Inf Syst. 4(1), 5-24 (2010)

6. M Chen, S Gonzalez, V Leung, Applications and design issues of mobile agents in wireless sensor networks. IEEE Wireless Commun Mag. 14(6), 20-26 (2007)

7. PV Rickenbach, R Wattenhofer, Gathering correlated data in sensor networks, in Proceedings of the ACM Joint Workshop Foundations of Mobile Computing (DIALM-POMC '04) (2004)

8. B Krishnamachari, The impact of data aggregation in wireless sensor networks, in Proceedings of International Workshop on Distributed EventBased Systems, pp. 575-578 (2002)

9. A Goel, K Munagala, Balancing Steiner trees and shortest path trees online, in Proceedings of the 11th Annual ACM-SIAM Symposium, Discrete Algorithms (SODA '00) (2000)

10. H Luo, Y Liu, SK Das, Distributed algorithm for en route aggregation decision in wireless sensor networks. IEEE Trans Mobile Comput. 8(1), 1-13 (2009)

11. A Anandkumar, W Meng, T Lang, A Swami, Prize-collecting data for costperformance tradeoff distributed inference, in it IEEE INFOCOM, pp, 2150-2158 (2009)

12. A Wun, M Petrovic, $\mathrm{H}$ Jacobsen, A system for semantic data fusion in sensor networks, in DEBS '07 Proceedings of the 2007 Inaugural International Conference on Distributed Event-based Systems (2007)

13. WR Heinzelman, AP Chandrakasan, H Balakrishnan, An application specific protocol architecture for wireless microsensor networks. IEEE Trans Wireless Commun. 1(4), 660-670 (2002). doi:10.1109/TWC.2002.804190

14. X Wang, T Berger, Self-organizing redundancy-cellular architecture for wireless sensor networks, in 2005 IEEE Wireless Communications and Networking Conference, WCNC 2005: Broadband Wireless for the MassesReady for Take-off, 1945-1951 (2005)

15. $Y X u, J$ Heide, D Estrin, Geography-informed energy conservation for ad hoc routing, in Proceedings of the 7th Annual International Conference on Mobile Computing and Network, Italy (ACM, New York, 2001), pp. 70-78

16. K Lin, M Chen, X Ge, Adaptive reliable routing based on cluster hierarchy for wireless multimedia sensor networks. EURASIP J Wireless Commun Networking 2010, 1-11 (2010)

17. R Liu, G Rogers, S Zhou, Honeycomb architecture for energy conversation in wireless sensor networks, in Proceedings of the 2006 IEEE Global
Telecommunications Conference, San Francisco, USA (IEEE, New York, 2006), pp. 1-5

18. CY Chang, KP Shih, SC Lee, ZBP: A zone-based broadcasting protocol for wireless sensor networks, in Proceedings of the 18th IEEE International Conference on Advanced Information Networking and Applications, Fukuoka, Japan, (IEEE, New York, 2004), pp. 84-89

19. K Lin, KQ Li, WL Xue, YG Bi, A clustering hierarchy based on cellular topology for wireless sensor networks. Int J Comput Sci Eng. 5, 51-59 (2009)

20. $X W u, G$ Chen, Avoiding energy holes in wireless sensor networks with nonuniform node distribution. IEEE Trans Parallel Distributed Syst. 19(5), 710-720 (2008)

21. K Deray, S Simoff, Designing technology for visualisation of interactions on mobile devices. J Comput Sci Eng. 3(4), 218-237 (2009)

22. B Kim, S Kang, IEEE 802.15.4 MAC-based location-ID exchange protocol for realizing micro-cell connectionless location-awareness services. J Comput Sci Eng. 2(4), 412-427 (2008)

23. K Kim, S Uno, M Kim, Adaptive QoS mechanism for wireless mobile network. J Comput Sci Eng. 4(2), 153-172 (2010)

24. S Raazi, S Lee, A survey on key management strategies for different applications of wireless sensor networks. J Comput Sci Eng. 4(1), 23-51 (2010)

25. ZM Wang, S Basagni, E Melachrinoudis, C Petrioli, Exploiting sink mobility for maximizing sensor networks lifetime, in Proceedings of the 38th Hawail International Conference (IEEE Computer Society, Washington, 2005), pp. $287-295$

26. R Shah, S Roy, S Jain, W Brunette, Data mules: modeling a three-tier architecture for sparse sensor networks, in Proceedings of the IEEE Workshop on Sensor Network Protocols and Applications (IEEE Computer Society, Piscataway, 2003), pp. 30-41

27. F Wang, D Wang, JC Liu, Traffic-aware relay node deployment for data collection in wireless sensor networks, in SECON'09 Proceedings of the 6th Annual IEEE Communications Society Conference on Sensor, Mesh and Ad Hoc Communications and Networks, pp. 1-9 (2009)

28. S Gandham, M Dawande, R Prakash, Energy-efficient schemes for wireless sensor networks with multiple mobile base stations, in Proceedings of the IEEE GLOBECOM (IEEE Computer Society, Washington, 2003), pp. 377-381

doi:10.1186/1687-1499-2011-142

Cite this article as: Lin and Chen: Research on energy efficient fusiondriven routing in wireless multimedia sensor networks. EURASIP Journal on Wireless Communications and Networking 2011 2011:142.

\section{Submit your manuscript to a SpringerOpen ${ }^{\mathcal{O}}$ journal and benefit from:}

- Convenient online submission

- Rigorous peer review

- Immediate publication on acceptance

- Open access: articles freely available online

- High visibility within the field

- Retaining the copyright to your article

Submit your next manuscript at $\gg$ springeropen.com 